\title{
Skeletal Muscle Loss during Tyrosine Kinase Inhibitor Treatment for Advanced Hepatocellular Carcinoma Patients
}

\author{
Shinsuke Uchikawa Tomokazu Kawaoka Maiko Namba \\ Kenichiro Kodama Kazuki Ohya Kei Morio Takashi Nakahara \\ Eisuke Murakami Masataka Tsuge Akira Hiramatsu Michio Imamura \\ Shoichi Takahashi Kazuaki Chayama Hiroshi Aikata \\ Department of Gastroenterology and Metabolism, Applied Life Sciences, Institute of \\ Biomedical \& Health Sciences, Hiroshima University, Hiroshima, Japan
}

\section{Keywords}

Skeletal muscle $\cdot$ Hepatocellular carcinoma $\cdot$ Tyrosine kinase inhibitor $\cdot$ Sorafenib $\cdot$ Lenvatinib

\begin{abstract}
Introduction: The measurement of body composition such as the skeletal muscle index (SMI) has been reported to be useful for predicting prognosis in hepatocellular carcinoma (HCC). In this study, we analyzed skeletal muscle change during sorafenib and lenvatinib therapy and the association between SMI and prognosis. Methods: A total of 67 patients with advanced HCC and Child-Pugh grade A status treated with tyrosine kinase inhibitors (TKIs) at Hiroshima University between September 2009 and December 2018 were enrolled in this retrospective cohort study. Patients underwent computed tomography (CT) imaging before starting sorafenib treatment and 1-3 months after treatment initiation. Results: In all patients, the median SMI was $45.3 \mathrm{~cm}^{2} / \mathrm{m}^{2}$ before TKI treatment and $42.1 \mathrm{~cm}^{2} / \mathrm{m}^{2}$ after treatment; 54 of 67 (80.6\%) patients experienced SMI loss. The median $\Delta$ SMI was $-1.5 \mathrm{~cm}^{2} / \mathrm{m}^{2} /$ months, and no difference in $\Delta S M I$ was observed between patients receiving sorafenib and lenvatinib. No significant differences were observed in median $\triangle S M I$ between patients with and without progressive disease $\left(-2.35\right.$ and $-1.1 \mathrm{~cm}^{2} / \mathrm{m}^{2} /$ months, respectively), albumin-bilirubin grade 1 and 2 group disease $\left(-1.7\right.$ and $-1.5 \mathrm{~cm}^{2} / \mathrm{m}^{2} /$ months, respectively), and relative dose intensity $\leq 80$ and $>80\left(-1.8\right.$ and $-1.2 \mathrm{~cm}^{2} / \mathrm{m}^{2} /$ months, respectively). Conclusion: This report demonstrated that patients receiving TKI treatment experienced a significant loss of skeletal muscle mass regardless of disease progression, hepatic reserve, or which TKI (sorafenib or lenvatinib) they received.




\section{Liver Cancer}

\section{Introduction}

Hepatocellular carcinoma (HCC) is one of the leading causes of cancer-related death worldwide [1]. HCC commonly occurs in patients with chronic hepatitis or liver cirrhosis due to hepatitis B virus (HBV) or hepatitis C virus (HBC), alcohol use, nonalcoholic steatohepatitis, or diabetes [2].

Tyrosine kinase inhibitors (TKIs) are currently the standard of care for the systemic treatment of patients with advanced HCC who are not candidates for potential curative options, such as surgical resection, transplantation, or locoregional therapy. Sorafenib and lenvatinib inhibit tumor cell proliferation and tumor angiogenesis-driven cancer growth [3-5].

Previous studies on prognostic factors in patients with HCC who underwent sorafenib therapy have been focused on tumor-associated factors, such as hepatic reserve, serum biomarkers, and combination therapy [6-8]. Interestingly, the measurement of body composition, such as skeletal muscle index (SMI), has also been reported to be useful for predicting prognosis [9-14]. Computed tomography (CT) is used to evaluate skeletal muscle volume.

In this retrospective study, we analyzed skeletal muscle change during sorafenib and lenvatinib therapy and the association between SMI and prognosis.

\section{Materials and Methods}

Patients

A total of 67 patients with advanced HCC and Child-Pugh grade A status who initiated sorafenib or lenvatinib as the first-line TKI therapy at Hiroshima University between September 2009 and December 2018 were enrolled in this retrospective cohort study. Patients underwent CT imaging prior to and 1-3 months or longer after treatment initiation.

\section{Treatment}

Patients received $400 \mathrm{mg}$ sorafenib twice daily or $8 \mathrm{mg} / 12 \mathrm{mg}$ lenvatinib once daily based on their body weight. Treatment interruptions and dose reductions were permitted for adverse drug reactions. Treatment continued until death or until one of the following criteria were met: (i) adverse events that required termination of treatment; (ii) deterioration of Eastern Cooperative Oncology Group performance status (ECOGPS); (iii) worsening liver function; (iv) withdrawal of consent. In our hospital, patients continued sorafenib and lenvatinib after the onset of radiological progressive disease (PD) as defined by the modified Response Evaluation Criteria in Solid Tumors (mRECIST) when no definitive treatment exists for second-line therapy. Criteria for treatment discontinuation after PD were severe adverse effects leading to discontinuation, symptomatic progression, deterioration of hepatic reserve, deterioration of ECOG PS, and death.

Assessment of Skeletal Muscle

All patients underwent CT before TKI treatment and 1-3 months afterwards (or longer). The skeletal muscle area was measured from CT images. Using sliceOmatic software v5.0 (Tomovision, Montreal, QC, Canada), cross-sectional CT images were analyzed at the level of the third lumbar vertebra (L3) to determine skeletal muscle area. Skeletal muscle was identified and quantified by thresholds of -29 to +150 Hounsfield units (HU) [15]. Skeletal muscle mass was normalized for height in $\mathrm{m}^{2}$ and expressed as the SMI in $\mathrm{cm}^{2} / \mathrm{m}^{2}$. Using SMI values, muscle depletion was defined as $<42 \mathrm{~cm}^{2} / \mathrm{m}^{2}$ in men and $<38 \mathrm{~cm}^{2} / \mathrm{m}^{2}$ in women, according to the Japan Society of Hepatology criteria [16]. Estimated SMI change during TKI therapy was based on the $\Delta$ SMI: (post SMI - pre SMI)/months from initiation to evaluation.

Evaluation of Response to Sorafenib and Lenvatinib

Radiological response was evaluated by CT 1-3 months or longer after sorafenib and lenvatinib initiation using mRECIST. Adverse drug reactions were defined according to the Common Terminology Criteria for Adverse Events v4.0 (CTCAE 4.0). 
Table 1. Patients' pretreatment characteristics

\begin{tabular}{|c|c|c|c|c|}
\hline & All $(n=67)$ & Sorafenib $(n=49)$ & Lenvatinib $(n=18)$ & $p$ value \\
\hline Age, years & $70(20-87)$ & $68.0(20-87)$ & $71.5(46-83)$ & 0.213 \\
\hline Gender, M/F & $56 / 11$ & $41 / 8$ & $15 / 3$ & 1 \\
\hline Etiology, HBV/HCV/NBNC/HBV + HCV & $16 / 28 / 22 / 1$ & $14 / 21 / 13 / 1$ & $2 / 7 / 9 / 0$ & 0.233 \\
\hline Prothrombin activity, \% & $85(45.0-106)$ & $89.0(45.0-106)$ & $84.5(63.5-99)$ & 0.295 \\
\hline Serum albumin, $\mathrm{g} / \mathrm{dL}$ & $3.9(2.9-4.8)$ & $3.9(3.1-4.8)$ & $3.6(2.9-4.8)$ & 0.098 \\
\hline Serum total bilirubin, $\mathrm{mg} / \mathrm{dL}$ & $0.8(0.4-1.7)$ & $0.8(0.4-1.7)$ & $0.8(0.4-1.7)$ & 0.775 \\
\hline Child-Pugh score $5 / 6$ & $44 / 23$ & $35 / 14$ & $9 / 9$ & 0.147 \\
\hline Serum alpha fetoprotein, $\mathrm{ng} / \mathrm{mL}$ & $46(0.5-2,650,000)$ & $46.0(1.2-2,650,000)$ & $52.3(0.5-121,590)$ & 0.921 \\
\hline Serum des- $\gamma$-carboxy prothrombin, $\mathrm{mAU} / \mathrm{mL}$ & $475(0.5-344,570)$ & $475(0.5-344,570)$ & $635(14.0-108,390)$ & 0.651 \\
\hline T stage $^{\mathrm{a}} 0 / 1 / 2 / 3 / 4$ & $12 / 3 / 16 / 23 / 13$ & $10 / 3 / 7 / 20 / 9$ & $2 / 0 / 9 / 3 / 4$ & 0.49 \\
\hline Extra hepatic metastasis absent/present & $26 / 41$ & $16 / 33$ & $10 / 8$ & 0.1 \\
\hline Size of hepatic tumor, $\mathrm{mm}$ & $23(0-180)$ & $26.6(0-180)$ & $19.5(0-120)$ & 0.599 \\
\hline Tumor size relative to the liver, $\leq 50 \% />50 \%$ & $57 / 10$ & $40 / 9$ & $17 / 1$ & 0.267 \\
\hline No. of hepatic tumors, $\leq 3 / \geq 4$ & $36 / 31$ & $29 / 20$ & $7 / 11$ & 0.173 \\
\hline MVI absent/present & $47 / 20$ & $34 / 15$ & $13 / 5$ & 1 \\
\hline L3-SMI, $\mathrm{cm}^{2} / \mathrm{m}^{2}$ & $45.3(26.6-62.0)$ & $44.6(26.6-62.0)$ & $45.7(37.3-61.4)$ & 0.876 \\
\hline Muscle depletion absent/present & $49 / 18$ & $35 / 14$ & $14 / 4$ & 0.76 \\
\hline
\end{tabular}

Data are expressed as median (range) or $n$. HBV, hepatitis B virus; HCV, hepatitis C virus; NBNC, non-hepatitis B virus and non-hepatitis C virus; MVI, macroscopic vascular invasion. ${ }^{a}$ Based on the following 3 conditions (T factor): solitary, $<2 \mathrm{~cm}$ in diameter, and no vessel invasion. T1 was defined as fulfilling all 3 conditions, T2 as fulfilling 2 of the conditions, T3 as fulfilling 1 of the conditions, and T4 as fulfilling none of the conditions.

\section{Statistical Analysis}

Univariate analysis was performed by log-rank test, using Fisher's exact test, the $\chi^{2}$ test, or the MannWhitney U test. The Wilcoxon rank sum test was used to compare differences in SMI before and after treatment. Multivariate analysis of prognostic factors was performed by Cox proportional hazards regression. Variables were identified as being significant if they had $p$ values $<0.05$ on univariate analysis. All statistical analyses were performed with EZR (Saitama Medical Center, Jichi Medical University, Saitama, Japan), a graphical user interface for $R$ (The $R$ Foundation for Statistical Computing, Vienna, Austria). More precisely, EZR is a modified version of $R$ commander designed to add the statistical functions frequently used in biostatistics. Both overall survival (OS) and change of skeletal muscle mass during TKI treatment were assessed.

\section{Results}

\section{Patients' Background Characteristics}

Patients' characteristics and clinical data at TKI initiation are summarized in Table 1 . The median patient age was 70 (range $20-87$ ) years and 56/67 patients (83.6\%) were male. Of all 67 patients, 16 (23.9\%) were HBV antigen-positive, and 28 (41.8\%) were HCV antibodypositive. Twenty patients (29.9\%) had macroscopic vascular invasion (MVI) and $41(61.2 \%)$ had extrahepatic metastasis. The median L3 SMI (L3-SMI) was 45.3 (range, 26.6-62.0) cm²/ $\mathrm{m}^{2}$.

\section{Assessment of the Response to TKI Treatment}

Based on mRECIST, the number of patients with PD and non-PD was 22 (sorafenib, 18; lenvatinib, 4) and 45 (sorafenib, 31; lenvatinib, 14), respectively, at the evaluation at 1-3 months. Figure 1 shows the median survival time (MST) for all patients (15.6 months), 


\section{Liver Cancer}

\begin{tabular}{l|l}
\hline Liver Cancer 2020;9:148-155 \\
\hline DOI: 10.1159/000503829 & $\begin{array}{l}\text { @ 2019 The Author(s). Published by S. Karger AG, Basel } \\
\text { www.karger.com/lic }\end{array}$ \\
\hline
\end{tabular}

Uchikawa et al.: Skeletal Muscle Loss during TKI Treatment for Advanced HCC

Table 2. Univariate and multivariate analysis of prognostic factors

\begin{tabular}{|c|c|c|c|c|}
\hline & $\begin{array}{l}\text { Univariate } \\
\text { analysis }^{\text {a }}\end{array}$ & $\begin{array}{l}\text { Multivariate } \\
\text { analysis }^{\mathrm{b}}\end{array}$ & $95 \% \mathrm{CI}$ & HR \\
\hline Age, years ( $\leq 70$ vs. $>70)$ & 0.112 & & & \\
\hline Gender (male vs. female) & 0.731 & & & \\
\hline Etiology (viral vs. NBNC) & 0.678 & & & \\
\hline Prothrombin activity, $\%(\leq 85$ vs. $>85)$ & 0.655 & & & \\
\hline Serum albumin, g/dL ( $\leq 3.9$ vs. $>3.9)$ & 0.331 & & & \\
\hline Serum total bilirubin, $\mathrm{mg} / \mathrm{dL}(\leq 0.8$ vs. $>0.8$ ) & 0.546 & & & \\
\hline Child-Pugh score (5 vs. 6 ) & 0.639 & & & \\
\hline ALBI grade (1 vs. 2 ) & 0.811 & & & \\
\hline Serum $\alpha$-fetoprotein, $\mathrm{ng} / \mathrm{mL}(\leq 46$ vs. $>46)$ & 0.113 & & & \\
\hline Serum des- $\gamma$-carboxy prothrombin, $\mathrm{mAU} / \mathrm{mL}(\leq 475$ vs. 475$)$ & $<0.001$ & 0.993 & $1.000-1.000$ & 1.0 \\
\hline Extrahepatic metastasis (absent/present) & 0.498 & & & \\
\hline Tumor size relative to the liver $(\leq 50$ vs. $>50)$ & 0.052 & & & \\
\hline Number of hepatic tumors ( $<4$ vs. $\geq 4$ ) & 0.026 & 0.286 & $0.7386-2.795$ & 1.4 \\
\hline Macroscopic vascular invasion (absent vs. present) & $<0.001$ & 0.001 & $1.657-8.332$ & 3.7 \\
\hline Muscle depletion (absent vs. present) & 0.437 & & & \\
\hline Relative dose intensity ( $\leq 80$ vs. $>80$ ) & 0.333 & & & \\
\hline mRECIST (PD vs. non-PD at first evaluation) & 0.122 & & & \\
\hline
\end{tabular}

CI, confidence interval; HR, hazard ratio; NBNC, non-hepatitis B virus and non-hepatitis C virus; ALBI, albumin-bilirubin; mRECIST, modified Response Criteria in Solid Tumors; PD, progressive disease; non-PD, nonprogressive disease. ${ }^{\text {a }}$ Log-rank test; ${ }^{\mathrm{b}}$ Cox proportional hazards regression.

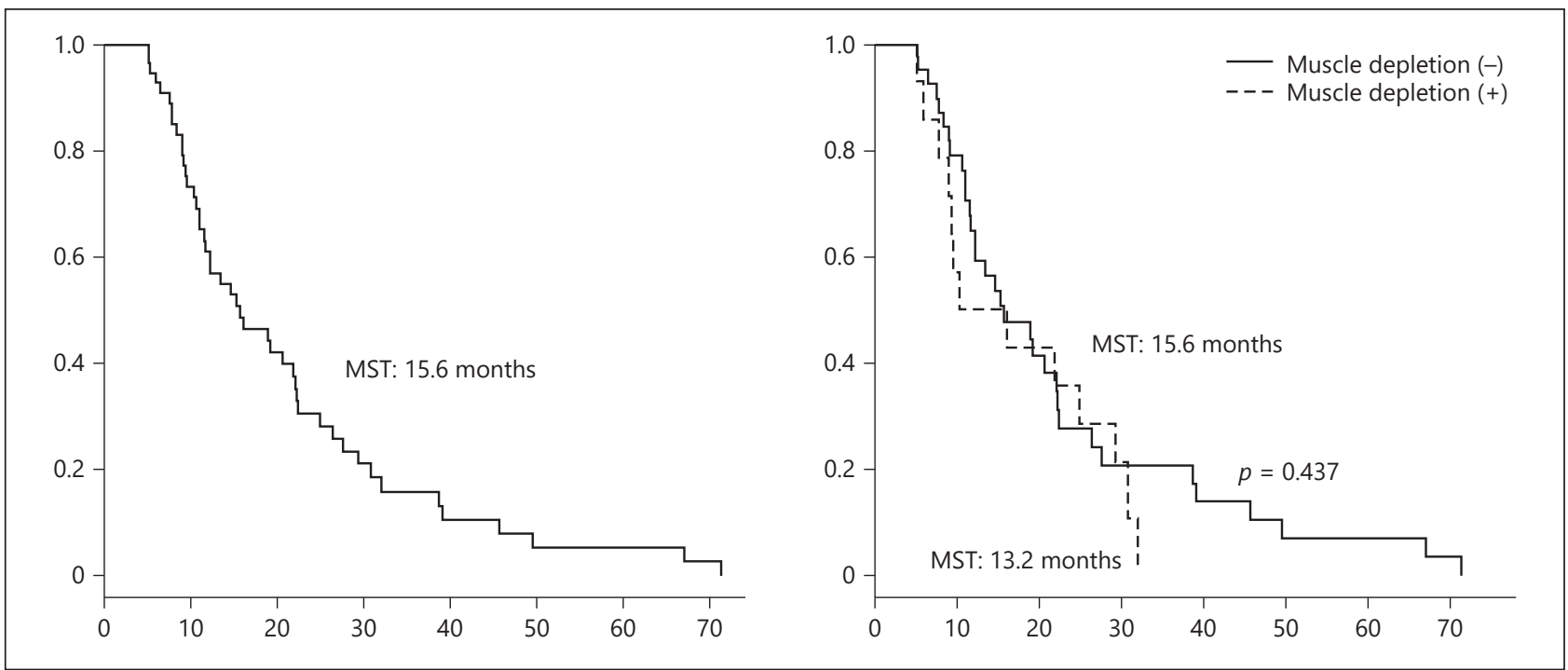

Fig. 1. Comparison of overall survival between patients with and without muscle depletion. MST, median survival time.

patients with muscle depletion (13.2 months), and patients without muscle depletion (15.6 months); the difference between these groups was not significant ( $p=0.437)$. Prognostic factors identified on univariate analysis included low serum des- $\gamma$-carboxy prothrombin (DCP), a small number of hepatic tumors, and an absence of MVI. Multivariate analysis revealed only MVI as a prognostic factor (Table 2). 


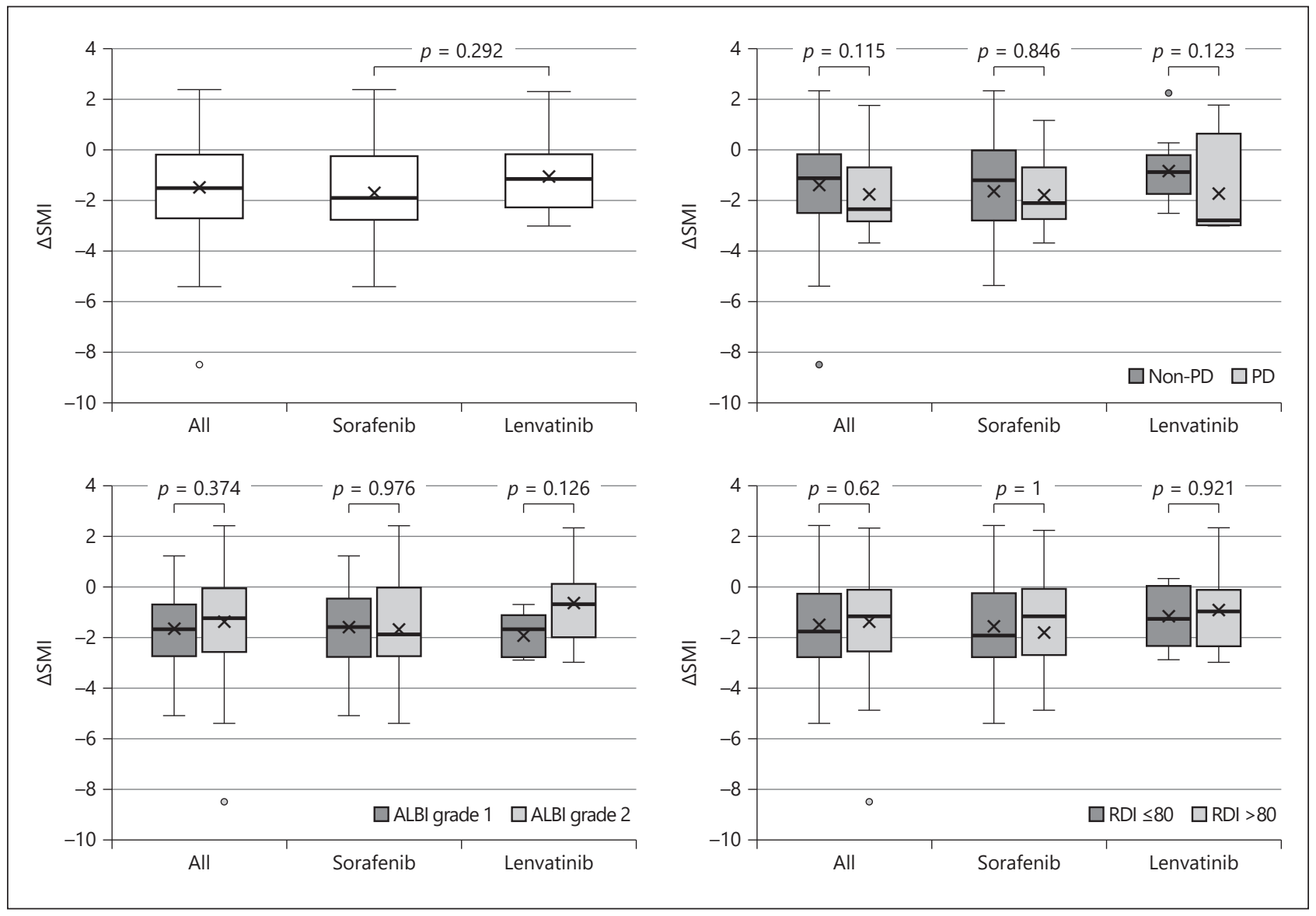

Fig. 2. Comparison of $\Delta \mathrm{SMI}$ between sorafenib and lenvatinib. The Mann-Whitney U test was used.

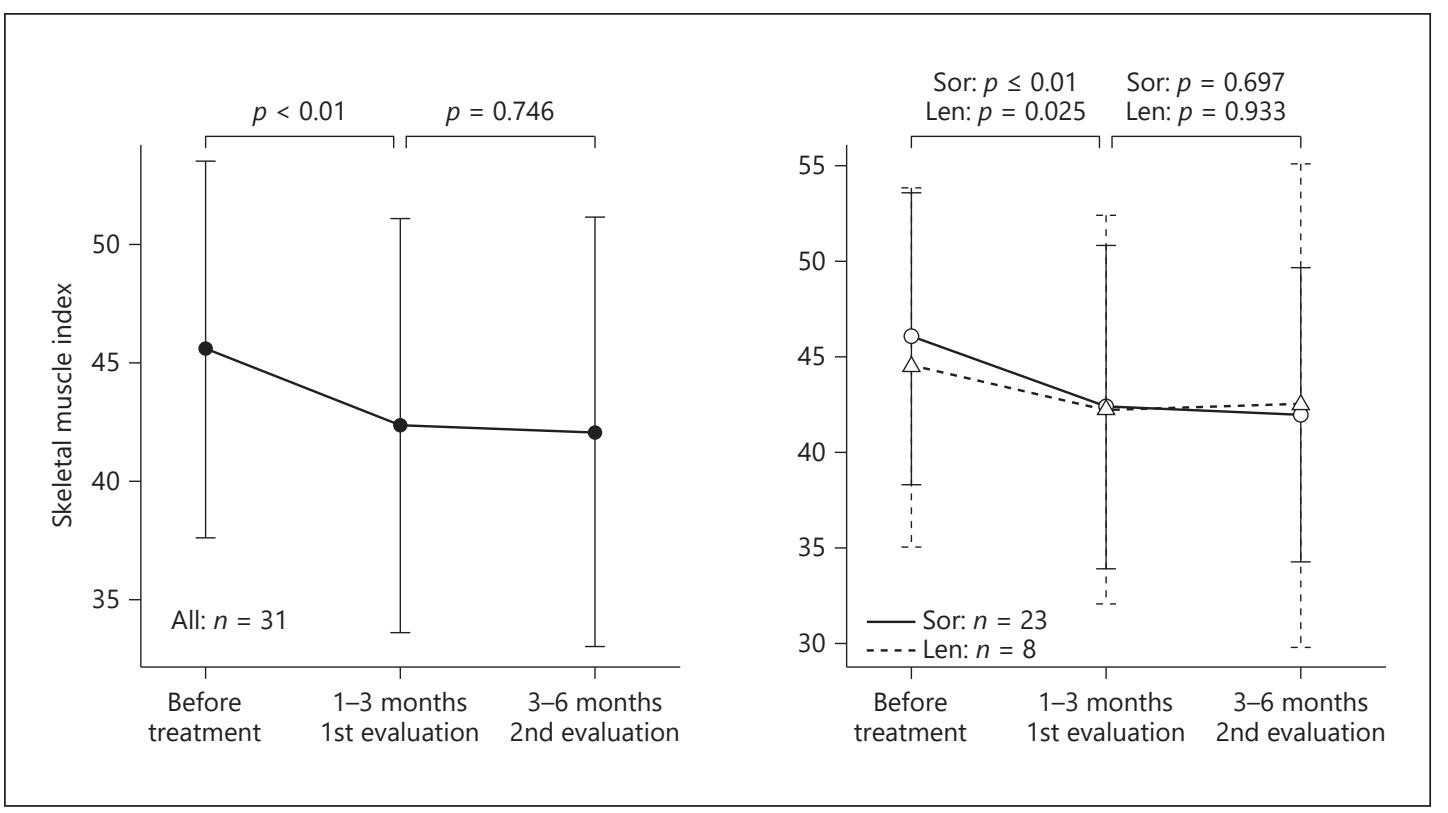

Fig. 3. Skeletal muscle change of patients was evaluated 3 times. Len, lenvatinib; Sor, sorafenib. The Wilcoxon rank sum test was used. 


\section{Liver Cancer}

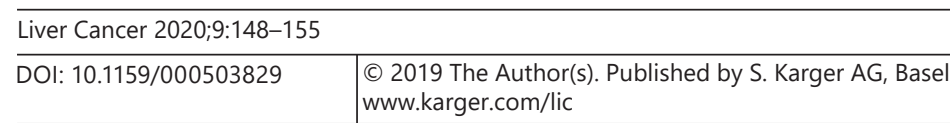

Uchikawa et al.: Skeletal Muscle Loss during TKI Treatment for Advanced HCC

\section{Skeletal Muscle Mass Changes in All Patients}

Figure 2 shows the evaluation of $\triangle$ SMI during treatment. In all patients, the median SMI before TKI treatment was $45.3 \mathrm{~cm}^{2} / \mathrm{m}^{2}$ and afterwards it was $42.1 \mathrm{~cm}^{2} / \mathrm{m}^{2} ; 54 / 67(80.6 \%)$ patients experienced a decrease in SMI.

\section{Skeletal Muscle Mass Changes by Subgroup}

The median $\Delta$ SMI was $-1.5 \mathrm{~cm}^{2} / \mathrm{m}^{2} /$ months, and no significant difference in $\Delta$ SMI was observed between the sorafenib and lenvatinib groups $(p=0.292)$. The median $\Delta$ SMI of patients with PD and non-PD was -2.35 and $-1.1 \mathrm{~cm}^{2} / \mathrm{m}^{2} /$ months, respectively $(p=0.115)$; between patients with albumin-bilirubin (ALBI) grade 1 and 2 disease it was -1.7 and -1.5 $\mathrm{cm}^{2} / \mathrm{m}^{2} /$ months, respectively $(p=0.374)$; and between patients who received a relative dose intensity (RDI) of $\leq 80$ and $>80$, it was -1.8 and $-1.2 \mathrm{~cm}^{2} / \mathrm{m}^{2} /$ months, respectively $(p=0.62)$. None of these differences was significant.

All patients showed a tendency to experience decreased skeletal muscle mass during TKI therapy. Figure 3 shows $\Delta$ SMI in 31 patients evaluated 3 times. A significant difference $(p<$ 0.01 ) was observed between baseline and the first evaluation, but no significant difference ( $p=0.746)$ was observed between the first and second evaluations. The result was similar in both the sorafenib and lenvatinib groups.

\section{Discussion}

In this study, we assessed the change of skeletal muscle mass in patients with HCC treated with TKIs. We used L3-SMI to evaluate the quantity of skeletal muscle and found that L3-SMI decreased in 54 of 67 patients treated with TKI therapy.

Several factors are associated with skeletal muscle depletion. In patients with cancer, reasons for skeletal muscle depletion include decreased physical activity and poor nutrition due to disease progression and the adverse effects of treatment, as well as increased expression of inflammatory cytokines [17]. In contrast, in patients with chronic liver disease, poor nutrition and loss of branch-chain amino acids (BCAAs) $[18,19]$ and carnitine $[20]$ contribute to skeletal muscle loss.

The median $\Delta$ SMI of patients with PD and non-PD was -2.35 and -1.1 , respectively. This suggests that patients treated with TKIs lose skeletal muscle regardless of tumor progression. When patients with ALBI grade 1 and 2 disease were compared, L3-SMI tended to decrease in both groups, and no difference in $\triangle$ SMI was observed. Moreover, L3-SMI decreased in patients who received sorafenib and in those who received lenvatinib. Previous reports have also demonstrated skeletal muscle loss in patients with renal-cell cancer [21] and lung cancer [22].

Sarcopenia is thought to be associated with alterations in the phosphoinositide PI3k/ Akt/mTOR signaling pathway [23-26]. This pathway is associated with muscle protein synthesis. Since sorafenib and lenvatinib both inhibit VEGFR-mediated signaling and the carnitine transporter [27], this may lead to the suppression of downstream PI3k/Akt/mTOR pathway signaling and a subsequent skeletal muscle decrease.

We also assessed prognostic factors in this study. A previous report stated that muscle depletion was a prognostic factor for patients with HCC [9-14]. In our study, DCP, the number of intrahepatic tumors, and MVI were identified as prognostic factors in univariate analysis, while multivariate analysis revealed only MVI as a prognostic factor. Muscle depletion was not associated with prognosis; however, this needs to be assessed in more patients, and a high rate of MVI (29.9\%) could also affect this result.

This report has some limitations, such as the small sample size, retrospective design, and short-term nature. We have shown that patients with TKI treatment significantly lost their 
Uchikawa et al.: Skeletal Muscle Loss during TKI Treatment for Advanced HCC

skeletal muscle mass regardless of disease progression, hepatic reserve, and TKI administration (either sorafenib or lenvatinib). From these results and those of previous reports [9-14], we should pay attention to muscle and nutrition during TKI therapy.

\section{Statement of Ethics}

This study was conducted in accordance with the ethics principles of the Declaration of Helsinki and was approved by the Institutional Review Board of Hiroshima University (IRB No. 695). Written informed consent was obtained from each patient after a detailed explanation of the study procedure.

\section{Disclosure Statement}

The authors declare that they have no conflict of interest.

\section{Funding Sources}

This work received no funding support.

\section{Author Contributions}

S. Uchikawa and T. Kawaoka designed the study, analyzed the data, and wrote the paper; S. Uchikawa acquired the data and performed the statistical analysis; T. Kawaoka and H. Aikata reviewed the results; $\mathrm{H}$. Aikata revised the manuscript for important intellectual content.

\section{References}

1 Bruix J, Gores GJ, Mazzaferro V. Hepatocellular carcinoma: clinical frontiers and perspectives. Gut. 2014 May; 63(5):844-55.

2 Singal AG, El-Serag HB. Hepatocellular carcinoma from epidemiology to prevention: translating knowledge into practice. Clin Gastroenterol Hepatol. 2015 Nov;13(12):2140-51.

3 Wilhelm SM, Carter C, Tang L, Wilkie D, McNabola A, Rong H, et al. BAY 43-9006 exhibits broad spectrum oral antitumor activity and targets the RAF/MEK/ERK pathway and receptor tyrosine kinases involved in tumor progression and angiogenesis. Cancer Res. 2004 Oct;64(19):7099-109.

4 Chang YS, Adnane J, Trail PA, Levy J, Henderson A, Xue D, et al. Sorafenib (BAY 43-9006) inhibits tumor growth and vascularization and induces tumor apoptosis and hypoxia in RCC xenograft models. Cancer Chemother Pharmacol. 2007 Apr;59(5):561-74.

5 Kudo M, Finn RS, Qin S, Han KH, Ikeda K, Piscaglia F, et al. Lenvatinib versus sorafenib in first-line treatment of patients with unresectable hepatocellular carcinoma: a randomised phase 3 non-inferiority trial. Lancet. 2018 Mar;391(10126):1163-73.

6 Llovet JM, Peña CE, Lathia CD, Shan M, Meinhardt G, Bruix J; SHARP Investigators Study Group. Plasma biomarkers as predictors of outcome in patients with advanced hepatocellular carcinoma. Clin Cancer Res. 2012 Apr;18(8):2290-300.

7 Ogasawara S, Chiba T, Ooka Y, Suzuki E, Kanogawa N, Saito T, et al. Liver function assessment according to the Albumin-Bilirubin (ALBI) grade in sorafenib-treated patients with advanced hepatocellular carcinoma. Invest New Drugs. 2015 Dec;33(6):1257-62.

8 Choi GH, Shim JH, Kim MJ, Ryu MH, Ryoo BY, Kang YK, et al. Sorafenib alone versus sorafenib combined with transarterial chemoembolization for advanced-stage hepatocellular carcinoma: results of propensity score analyses. Radiology. 2013 Nov;269(2):603-11.

9 Prado CM, Lieffers JR, McCargar LJ, Reiman T, Sawyer MB, Martin L, et al. Prevalence and clinical implications of sarcopenic obesity in patients with solid tumours of the respiratory and gastrointestinal tracts: a population-based study. Lancet Oncol. 2008 Jul;9(7):629-35.

10 Nishikawa H, Nishijima N, Enomoto H, Sakamoto A, Nasu A, Komekado H, et al. Prognostic significance of sarcopenia in patients with hepatocellular carcinoma undergoing sorafenib therapy. Oncol Lett. 2017 Aug; 14(2):1637-47. 
Uchikawa et al.: Skeletal Muscle Loss during TKI Treatment for Advanced HCC

11 Imai K, Takai K, Hanai T, Ideta T, Miyazaki T, Kochi T, et al. Skeletal muscle depletion predicts the prognosis of patients with hepatocellular carcinoma treated with sorafenib. Int J Mol Sci. 2015 Apr;16(5):9612-24.

12 Saeki I, Yamasaki T, Maeda M, Kawano R, Hisanaga T, Iwamoto T, et al. No Muscle Depletion with High Visceral Fat as a Novel Beneficial Biomarker of Sorafenib for Hepatocellular Carcinoma. Liver Cancer. 2018 Oct; 7(4): 359-71.

13 Martin L, Birdsell L, Macdonald N, Reiman T, Clandinin MT, McCargar LJ, et al. Cancer cachexia in the age of obesity: skeletal muscle depletion is a powerful prognostic factor, independent of body mass index. J Clin Oncol. 2013 Apr;31(12):1539-47.

14 Hiraoka A, Hirooka M, Koizumi Y, Izumoto H, Ueki H, Kaneto M, et al. Muscle volume loss as a prognostic marker in hepatocellular carcinoma patients treated with sorafenib. Hepatol Res. 2017 May;47(6):558-65.

15 Mitsiopoulos N, Baumgartner RN, Heymsfield SB, Lyons W, Gallagher D, Ross R. Cadaver validation of skeletal muscle measurement by magnetic resonance imaging and computerized tomography. J Appl Physiol (1985). 1998 Jul;85(1):115-22.

16 Nishikawa H, Shiraki M, Hiramatsu A, Moriya K, Hino K, Nishiguchi S. Japan Society of Hepatology guidelines for sarcopenia in liver disease (1st edition): Recommendation from the working group for creation of sarcopenia assessment criteria. Hepatol Res 2016; 46: 951-63

17 Fujiwara N, Nakagawa H, Kudo Y, Tateishi R, Taguri M, Watadani T, et al. Sarcopenia, intramuscular fat deposition, and visceral adiposity independently predict the outcomes of hepatocellular carcinoma. J Hepatol. 2015 Jul;63(1):131-40.

18 Dasarathy S. Cause and management of muscle wasting in chronic liver disease. Curr Opin Gastroenterol. 2016 May;32(3):159-65.

19 Sinclair M, Gow PJ, Grossmann M, Angus PW. Review article: sarcopenia in cirrhosis-aetiology, implications and potential therapeutic interventions. Aliment Pharmacol Ther. 2016 Apr;43(7):765-77.

20 Ringseis R, Keller J, Eder K. Mechanisms underlying the anti-wasting effect of L-carnitine supplementation under pathologic conditions: evidence from experimental and clinical studies. Eur J Nutr. 2013 Aug;52(5): 1421-42.

21 Antoun S, Birdsell L, Sawyer MB, Venner P, Escudier B, Baracos VE. Association of skeletal muscle wasting with treatment with sorafenib in patients with advanced renal cell carcinoma: results from a placebo-controlled study. J Clin Oncol. 2010 Feb;28(6):1054-60.

22 Kakinuma K, Tsuruoka H, Morikawa K, Furuya N, Inoue T, Miyazawa T, et al. Differences in skeletal muscle loss caused by cytotoxic chemotherapy and molecular targeted therapy in patients with advanced non-small cell lung cancer. Thorac Cancer. 2018 Jan;9(1):99-104.

23 Filbin MG, Dabral SK, Pazyra-Murphy MF, Ramkissoon S, Kung AL, Pak E, et al. Coordinate activation of Shh and PI3K signaling in PTEN-deficient glioblastoma: new therapeutic opportunities. Nat Med. 2013 Nov; 19(11):1518-23.

24 Benoit B, Meugnier E, Castelli M, Chanon S, Vieille-Marchiset A, Durand C, et al. Fibroblast growth factor 19 regulates skeletal muscle mass and ameliorates muscle wasting in mice. Nat Med. 2017 Aug;23(8):990-6.

25 Bravo-San Pedro JM, Kroemer G, Galluzzi L. Autophagy and Mitophagy in Cardiovascular Disease. Circ Res. 2017 May;120(11):1812-24.

26 Narita M, Young AR, Arakawa S, Samarajiwa SA, Nakashima T, Yoshida S, et al. Spatial coupling of mTOR and autophagy augments secretory phenotypes. Science. 2011 May;332(6032):966-70.

$27 \mathrm{Hu}$ C, Lancaster CS, Zuo Z, Hu S, Chen Z, Rubnitz JE, et al. Inhibition of OCTN2-mediated transport of carnitine by etoposide. Mol Cancer Ther. 2012 Apr;11(4):921-9. 